\title{
On the $L^{p}$ index of spin Dirac operators on conical manifolds
}

\author{
by \\ André Legrand (Toulouse) and Sergiu Moroianu (Bucureşti)
}

\begin{abstract}
We compute the index of the Dirac operator on a spin Riemannian manifold with conical singularities, acting from $L^{p}\left(\Sigma^{+}\right)$to $L^{q}\left(\Sigma^{-}\right)$with $p, q>1$. When $1+n / p-n / q>0$ we obtain the usual Atiyah-Patodi-Singer formula, but with a spectral cut at $(n+1) / 2-n / q$ instead of 0 in the definition of the eta invariant. In particular we reprove Chou's formula for the $L^{2}$ index. For $1+n / p-n / q \leq 0$ the index formula contains an extra term related to the Calderón projector.
\end{abstract}

1. Introduction. Let $\left(X, g_{\text {cone }}\right)$ be a spin Riemannian manifold with isolated conical singularities. The $L^{2}$ index of the Dirac operator $D_{\text {cone }}^{+}$on $X$ was computed in [3]. In this paper we first derive Chou's formula from the index formula of Atiyah, Patodi and Singer [1]. The viewpoint of this paper is that the passage between the two problems is actually elementary. We then use our method to compute the index of the Dirac operator from $L^{p}$ to $L^{q}$.

TheOREM 1. Let $\left(X, g_{\text {cone }}\right)$ be a spin conical manifold of even dimension $n$, and $p, q>1$. Set $\alpha_{1}:=n / p-(n-1) / 2, \alpha_{2}:=(n+1) / 2-n / q$. Let

$$
D_{p, q, \text { cone }}^{+}: \mathcal{C}_{\mathrm{c}}^{\infty}\left(X, \Sigma^{+}\right) \subset L^{p}\left(X, \Sigma^{+}, g_{\text {cone }}\right) \rightarrow L^{q}\left(X, \Sigma^{-}, g_{\text {cone }}\right)
$$

be the chiral Dirac operator acting as an unbounded operator in Banach spaces. Then for $\alpha_{1}+\alpha_{2}=1+n / p-n / q>0$,

$$
\operatorname{index}\left(D_{p, q, \text { cone }}^{+}\right)=\int_{X} \widehat{A}\left(g_{\text {cone }}\right)+\frac{1}{2} \eta_{\left[\alpha_{2}, \infty\right)}(M),
$$

2000 Mathematics Subject Classification: Primary 58J20.

Key words and phrases: Dirac operator, spin conical manifold, index theorem, eta invariant.

We acknowledge the support of the Research and Training Networks HPRNCT-1999-00118 "Geometric Analysis" and HPRN-CT-2002-00280 "Quantum SpacesNoncommutative Geometry" funded by the European Commission. 
while for $1+n / p-n / q \leq 0$,

$$
\operatorname{index}\left(D_{p, q, \text { cone }}^{+}\right)=\int_{X} \widehat{A}\left(g_{\text {cone }}\right)+\frac{1}{2} \eta_{\left[-\alpha_{1}, \infty\right)}(M)-\operatorname{dim}\left(\mathcal{C}_{\left[\alpha_{2},-\alpha_{1}\right]}\right) .
$$

The necessary definitions are recalled in Section 2. The term $\mathcal{C}_{\left[\alpha_{2},-\alpha_{1}\right]}$ is a non-negative integer related to the Calderón projector. We immediately deduce Chou's result by setting $p=q=2$. Here are other corollaries:

Corollary 2. Let $p>1$ and $\alpha:=1 / 2+n / p-n / 2$, so $\alpha>0 \Leftrightarrow p<$ $2 n /(n-1)$. Let $p^{\prime} \in(1, \infty)$ be defined by $1 / p+1 / p^{\prime}=1$, so that the Dirac operator

$$
D_{p, p^{\prime}, \text { cone }}: \mathcal{C}_{\mathrm{c}}^{\infty}(X, \Sigma) \subset L^{p}\left(X, \Sigma, g_{\text {cone }}\right) \rightarrow L^{p^{\prime}}\left(X, \Sigma, g_{\text {cone }}\right)
$$

is symmetric. Then for $p<2 n /(n-1)$,

$$
\operatorname{index}\left(D_{p, p^{\prime}, \text { cone }}^{+}\right)=\int_{X} \widehat{A}\left(g_{\text {cone }}\right)+\frac{1}{2} \eta_{[\alpha, \infty)}(M),
$$

while for $p \geq 2 n /(n-1)$,

$$
\operatorname{index}\left(D_{p, p^{\prime}, \text { cone }}^{+}\right)=\int_{X} \widehat{A}\left(g_{\text {cone }}\right)+\frac{1}{2} \eta_{[-\alpha, \infty)}(M)-\operatorname{dim}\left(\mathcal{C}_{[\alpha,-\alpha]}\right) .
$$

Next we look at the Dirac operator acting on a fixed $L^{p}$. Cone operators in this context were considered by Schrohe and Seiler [7].

Corollary 3. Let $p>1$ and $\alpha:=(n+1) / 2-n / p$. Consider the Dirac operator

$$
D_{p, p, \text { cone }}^{+}: \mathcal{C}_{\mathrm{c}}^{\infty}\left(X, \Sigma^{+}\right) \subset L^{p}\left(X, \Sigma^{+}, g_{\text {cone }}\right) \rightarrow L^{p}\left(X, \Sigma^{-}, g_{\text {cone }}\right) .
$$

Then

$$
\operatorname{index}\left(D_{p, p, \text { cone }}^{+}\right)=\int_{X} \widehat{A}\left(g_{\text {cone }}\right)+\frac{1}{2} \eta_{[\alpha, \infty)}(M) .
$$

In particular, for $p=q=2 n /(n+1)$ the index of the Dirac operator has the same form as the APS index formula (Theorem 11) on manifolds with boundary.

Let us outline the proof of Theorem 1 . For simplicity consider the $L^{2}$ case. First, we conjugate $D_{\text {cone }}$ acting in $L^{2}\left(X, \Sigma, g_{\text {cone }}\right)$ to an unbounded operator in $L^{2}\left(X, \Sigma, g_{\text {cyl }}\right)$, where $g_{\text {cyl }}$ is a cylindrical metric on $X$ conformal to $g_{\text {cone }}$, thus transforming the problem to another $L^{2}$ index problem. Secondly, we relate this problem to the APS problem by restricting to a finite-length part of the cylinder. It turns out that the $L^{2}$ kernel and cokernel of the conical Dirac operator will be isomorphic to the kernel, respectively cokernel of an APS-type problem with a slightly different boundary spectral projection. This is easily related to the usual APS index formula, by counting the 
number of eigenvalues between 0 and $1 / 2$ of the boundary operator. Finally, we remark that the $\widehat{A}$ form is conformally invariant.

Earlier papers on $L^{2}$ cohomology and index for conical manifolds may have made the whole subject look forbiddingly technical. We would like to reassure the reader that this is not the case here. Except for the APS theorem which we take for granted, we give elementary proofs of all results. In particular we do not: construct heat kernels, parametrices, use pseudodifferential calculi or $L^{p}$ Sobolev spaces (though the $L^{2}$ Sobolev embeddings on a closed manifold must be used at one point).

Acknowledgments. The second author is grateful to Andrei Moroianu for useful discussions. He would also like to thank the Equipe de Géométrie Noncommutative de Toulouse for their warm hospitality at the Paul Sabatier University, where this paper was written.

\section{Background}

2.1. Conical manifolds. The fact that $\left(X, g_{\text {cone }}\right)$ is conical means that outside a compact set, $\left(X, g_{\text {cone }}\right)$ is isometric to $\left((0, \varepsilon) \times M, d r^{2}+r^{2} g_{M}\right)$, where $\left(M, g_{M}\right)$ is a compact, possibly disconnected Riemannian manifold.

ExAmple 4. Let $(\bar{X}, g)$ be a closed Riemannian manifold and $O \in \bar{X}$ a Euclidean point, in the sense that $g$ is flat in a neighborhood of $O$. In polar coordinates we see that $\bar{X} \backslash\{O\}$ is a conical manifold.

Such conical points are called fictitious, following Dines and Schulze [4]. They turn out to be interesting, and we treat them in Section 7.

2.2. Index in Banach spaces. Let $D^{+}: \operatorname{dom}\left(D^{+}\right) \subset H^{+} \rightarrow H^{-}$be a densely defined unbounded linear operator between Hilbert spaces, with densely defined adjoint $D^{+*}$. Let $\overline{D^{+}}$be the closure of $D^{+}$.

Definition 5. Assume that $\overline{D^{+}}$and $D^{+*}$ have finite-dimensional kernels. The index of $D^{+}$is defined by

$$
\operatorname{index}\left(D^{+}\right):=\operatorname{dim} \operatorname{ker} \overline{D^{+}}-\operatorname{dim} \operatorname{ker} D^{+*} \text {. }
$$

This is a generalization of the Fredholm index, in that the range of $\overline{D^{+}}$ need not be closed.

More generally, the definition holds for $H^{ \pm}$Banach spaces. For completeness and since it plays a crucial role below, we give the definition of $D^{+*}$ and $\overline{D^{+}}$.

Definition 6. Let $H^{ \pm}$be Banach spaces. Let $\operatorname{dom}\left(D^{+*}\right)$ be the space of those $u^{-} \in H^{-\prime}$ such that the map

$$
\operatorname{dom}\left(D^{+}\right) \ni \phi^{+} \mapsto u^{-}\left(D^{+} \phi^{+}\right)
$$


is bounded. Since $\operatorname{dom}\left(D^{+}\right)$is assumed to be dense, this map induces a bounded map on $H^{+}$. By the definition of the dual space, there exists a unique $u^{+} \in H^{+^{\prime}}$ so that $u^{-}\left(D^{+} \phi^{+}\right)=u^{+}\left(\phi^{+}\right)$for all $\phi^{+} \in \operatorname{dom}\left(D^{+}\right)$. We define $D^{+*} u^{-}:=u^{+}$.

Let $H:=H^{+} \oplus H^{-}$so $H^{\prime}=H^{+\prime} \oplus H^{-\prime}$. The graph of $D^{+}$is

$$
G\left(D^{+}\right):=\left\{\left(\phi, D^{+} \phi\right) ; \phi \in \operatorname{dom}\left(D^{+}\right)\right\} \subset H
$$

and similarly for $D^{+*}$. Define a bilinear pairing

$$
H \times H^{\prime} \rightarrow \mathbb{R}, \quad\left(\phi^{+}, \phi^{-}\right) \times\left(u^{+}, u^{-}\right) \mapsto u^{+}\left(\phi^{+}\right)-u^{-}\left(\phi^{-}\right) .
$$

Then $G\left(D^{+}\right) \perp G\left(D^{+*}\right)$ under this pairing. The pairing is continuous so $\overline{G\left(D^{+}\right)} \perp G\left(D^{+*}\right)$. Now we use the assumption that $\operatorname{dom}\left(D^{+*}\right)$ is dense (in practice this assumption is checked by constructing a formal adjoint with dense domain): it implies that a vector of the form $\left(\phi^{+}, 0\right)$ cannot belong to $\overline{G\left(D^{+}\right)}$unless $\phi^{+}=0$. So $\overline{G\left(D^{+}\right)}$is the graph of an operator.

Definition 7. $\overline{D^{+}}$is the operator with closed graph $\overline{G\left(D^{+}\right)}$.

Clearly, $\left(\overline{D^{+}}\right)^{*}=D^{+*}$. We define the index of $D^{+}$by Definition 5 . Note that $D^{+* *}=\overline{D^{+}}$if and only if $H^{ \pm}$are reflexive.

Specializing to the case of interest, let $(X, g)$ be a Riemannian manifold, $\Sigma^{ \pm}$hermitian vector bundles over $X, p, q>1$ and

$$
D^{+}: \mathcal{C}_{\mathrm{c}}^{\infty}\left(X, \Sigma^{+}\right) \subset L^{p}\left(X, \Sigma^{+}, g\right) \rightarrow L^{q}\left(X, \Sigma^{-}, g\right)
$$

a differential operator with formal adjoint

$$
D^{-}: \mathcal{C}_{\mathrm{c}}^{\infty}\left(X, \Sigma^{-}\right) \subset L^{q^{\prime}}\left(X, \Sigma^{-}, g\right) \rightarrow L^{p^{\prime}}\left(X, \Sigma^{+}, g\right),
$$

where $p^{\prime}$ is defined by $1 / p+1 / p^{\prime}=1$ and similarly for $q^{\prime}$. The elements of $L^{q^{\prime}}\left(X, \Sigma^{-}, g\right)$ act as distributions on $L^{q}\left(X, \Sigma^{-}, g\right)$. We define the distributional action of $D^{-}$on $u^{-} \in L^{q^{\prime}}\left(X, \Sigma^{-}, g\right)$ in the usual way:

$$
\mathcal{C}_{\mathrm{c}}^{\infty}\left(X, \Sigma^{+}\right) \ni \phi^{+} \mapsto\left(D^{-} u^{-}\right)\left(\phi^{+}\right):=u^{-}\left(D^{+} \phi^{+}\right) .
$$

Lemma 8. A section $u^{-} \in L^{q^{\prime}}\left(X, \Sigma^{-}, g\right)$ belongs to $\operatorname{dom}\left(D^{+*}\right)$ if and only if the distributional derivative $D^{-} u^{-}$belongs to $L^{p^{\prime}}\left(X, \Sigma^{+}, g\right)$. In that case, $D^{+*} u^{-}=D^{-} u^{-}$.

Proof. Immediate from the definition of $\operatorname{dom}\left(D^{+*}\right)$ and $D^{-} u^{-}$.

2.3. The eta invariant. Let $M$ be a compact spin manifold. For any interval $I \subset \mathbb{R}$, let $\Pi_{I}: \mathcal{C}^{\infty}(M, \Sigma(M)) \rightarrow \mathcal{C}^{\infty}(M, \Sigma(M))$ be the spectral projection associated to the Dirac operator $D_{M}$ and $I$; more precisely, if $\left(\phi_{\lambda}\right)$ is an eigenspinor of $D_{M}$ of eigenvalue $\lambda$, then

$$
\Pi_{I}\left(\phi_{\lambda}\right)= \begin{cases}\phi_{\lambda} & \text { if } \lambda \in I \\ 0 & \text { otherwise }\end{cases}
$$


Associated to $I$ we define the complex function

$$
\eta_{I}\left(D_{M}, z\right):=\operatorname{Tr}\left(\left(2 \Pi_{I}-1\right)\left(D_{M}^{2}\right)^{-z / 2}\right) .
$$

Special care is needed for the eigenvalue 0; we simply define the complex powers to be 1 on the nullspace of $D_{M}$. The generalized eta function $\eta_{I}$ is well defined and holomorphic for $\Re(z)>\operatorname{dim}(M)$ and extends meromorphically to $\mathbb{C}$ with simple poles. When $I=\mathbb{R}$ we get the zeta function of $D_{M}$; when $I=[0, \infty)$ we get the extended eta function of [1]. The function $\eta_{I}$ is always regular at $z=0$ (see [1]). We denote this regular(ized) value by $\eta_{I}(M)$.

Lemma 9. For $\alpha<\beta$ we have

$$
\eta_{[\alpha, \infty)}(M)=\eta_{[\beta, \infty)}(M)+2 N[\alpha, \beta)
$$

where $N[\alpha, \beta)$ is the number of eigenvalues of $D_{M}$ (counted with multiplicity) in the interval $[\alpha, \beta)$.

Proof. Clearly,

$$
\eta_{[\alpha, \infty)}\left(D_{M}, z\right)-\eta_{[\beta, \infty)}\left(D_{M}, z\right)=2 \sum_{\substack{\alpha \leq \lambda<\beta \\ \lambda \in \operatorname{Spec}\left(D_{M}\right)}} \lambda^{-z} .
$$

Recall that we defined $0^{z}=1$ for all $z$. Evaluating at $z=0$ we get the result.

2.4. The Atiyah-Patodi-Singer index formula. Let $Y$ be an even-dimensional compact spin manifold with boundary $M$, and $t: Y \rightarrow(-\infty, 0]$ (the negative of) a boundary-defining function. Fix a product decomposition $[-\varepsilon, 0] \times M \hookrightarrow Y$ of $Y$ near $M$, and a Riemannian metric on $Y$ which is of product type near the boundary:

$$
g=d t^{2}+g_{M}
$$

Over the cylinder $(-\varepsilon, 0] \times M$ there exist canonical isomorphisms of the spinor bundles $\Sigma^{ \pm}$with the spinor bundle of $M$ for the induced spin structure. With these identifications, we have

$$
D_{\text {cyl }}^{+}=\partial_{t}+D_{M}, \quad D_{\text {cyl }}^{-}=-\partial_{t}+D_{M},
$$

where $D_{M}$ is the Dirac operator on $M$. Set

$$
\mathcal{C}^{\infty}\left(Y, \Sigma^{ \pm}, \Pi_{I}\right):=\left\{\phi \in \mathcal{C}^{\infty}\left(Y, \Sigma^{ \pm}\right) ; \phi_{\mid M} \in \operatorname{Ran}\left(\Pi_{I}\right)\right\} .
$$

For $\alpha \in \mathbb{R}$ consider the Dirac operators (for the metric $g$ ) with spectral boundary conditions

$$
\begin{aligned}
& D_{\mathrm{APS}, \alpha}^{+}: \mathcal{C}^{\infty}\left(Y, \Sigma^{+}, \Pi_{[\alpha, \infty)}\right) \rightarrow \mathcal{C}^{\infty}\left(Y, \Sigma^{-}\right), \\
& D_{\mathrm{APS}, \alpha}^{-}: \mathcal{C}^{\infty}\left(Y, \Sigma^{-}, \Pi_{(-\infty, \alpha)}\right) \rightarrow \mathcal{C}^{\infty}\left(Y, \Sigma^{+}\right),
\end{aligned}
$$


and more generally for $I \subset \mathbb{R}$,

$$
D_{\mathrm{APS}, I}^{ \pm}: \mathcal{C}^{\infty}\left(Y, \Sigma^{ \pm}, \Pi_{I}\right) \rightarrow \mathcal{C}^{\infty}\left(Y, \Sigma^{\mp}\right) .
$$

These are continuous operators between Fréchet spaces. Being Fredholm in this context means having finite-dimensional kernel and co-image, which implies that the range is closed by the open mapping theorem.

REMARK 10. By integration by parts, (1) implies that ker $D_{\mathrm{APS}, \alpha}^{ \pm}$is orthogonal to $\operatorname{Ran}\left(D_{\mathrm{APS}, \alpha}^{\mp}\right)$ with respect to the $L^{2}$ inner product on $\mathcal{C}^{\infty}\left(Y, \Sigma^{ \pm}, g\right)$.

Theorem $11([1])$. The Dirac operators $D_{\mathrm{APS}, 0}^{ \pm}$are Fredholm,

$$
\operatorname{ker} D_{\mathrm{APS}, 0}^{ \pm}+\operatorname{Ran}\left(D_{\mathrm{APS}, 0}^{\mp}\right)=\mathcal{C}^{\infty}\left(Y, \Sigma^{ \pm}\right)
$$

and the index of $D_{\mathrm{APS}, 0}^{+}$is given by

$$
\operatorname{index}\left(D_{\mathrm{APS}, 0}^{+}\right)=\int_{Y} \widehat{A}(g)+\frac{1}{2} \widetilde{\eta}(M),
$$

where $\widehat{A}(g)$ is the Hirzebruch A-hat form, and $\widetilde{\eta}(M):=\eta_{[0, \infty)}(M)$ is the extended eta invariant of the operator $D_{M}$.

The explanation for the unusual sign in front of the eta invariant is our non-standard choice of sign for the variable $t$.

It is easy to deduce from this a similar result for $D_{\mathrm{APS}, \alpha}^{ \pm}$.

Corollary 12. For $\alpha \in \mathbb{R}$, the operator $D_{\mathrm{APS}, \alpha}^{+}$is Fredholm,

$$
\begin{aligned}
& \operatorname{ker} D_{\mathrm{APS}, \alpha}^{ \pm}+\operatorname{Ran}\left(D_{\mathrm{APS}, \alpha}^{\mp}\right)=\mathcal{C}^{\infty}\left(Y, \Sigma^{ \pm}\right), \\
& \operatorname{index}\left(D_{\mathrm{APS}, \alpha}^{+}\right)=\int_{Y} \widehat{A}(g)+\frac{1}{2} \eta_{[\alpha, \infty)}(M) .
\end{aligned}
$$

Proof. For simplicity assume $\alpha>0$. The evaluation map at the boundary

$$
\mathcal{C}^{\infty}\left(Y, \Sigma^{+}, \Pi_{[0, \infty)}\right) / \mathcal{C}^{\infty}\left(Y, \Sigma^{+}, \Pi_{[\alpha, \infty)}\right) \rightarrow \operatorname{Ran}\left(\Pi_{[0, \alpha)}\right)
$$

is an isomorphism, and by definition $\operatorname{dim}\left(\operatorname{Ran}\left(\Pi_{[0, \alpha)}\right)\right)=N[0, \alpha)$. Thus $D_{\mathrm{APS}, \alpha}^{+}$is the restriction of $D_{\mathrm{APS}, 0}^{+}$to a Fréchet subspace of codimension $N[0, \alpha)$. This shows (by Theorem 11) that $D_{\mathrm{APS}, \alpha}^{+}$has finite-dimensional index

$$
\operatorname{index}\left(D_{\mathrm{APS}, \alpha}^{+}\right)=\operatorname{index}\left(D_{\mathrm{APS}, 0}^{+}\right)-N[0, \alpha),
$$

which by Lemma 9 implies (3). Similarly

$$
\operatorname{index}\left(D_{\mathrm{APS}, \alpha}^{-}\right)=\operatorname{index}\left(D_{\mathrm{APS}, 0}^{-}\right)+N[0, \alpha),
$$

so in particular, from Theorem 11,

$$
\operatorname{index}\left(D_{\mathrm{APS}, \alpha}^{+}\right)=-\operatorname{index}\left(D_{\mathrm{APS}, \alpha}^{-}\right) .
$$


We have seen above (Remark 10) that $\operatorname{ker} D_{\mathrm{APS}, \alpha}^{ \pm} \perp \operatorname{Ran} D_{\mathrm{APS}, \alpha}^{\mp}$ so

$$
\begin{aligned}
& \operatorname{index}\left(D_{\mathrm{APS}, \alpha}^{+}\right) \leq \operatorname{dim} \operatorname{ker} D_{\mathrm{APS}, \alpha}^{+}-\operatorname{dim} \operatorname{ker} D_{\mathrm{APS}, \alpha}^{-}, \\
& \operatorname{index}\left(D_{\mathrm{APS}, \alpha}^{-}\right) \leq \operatorname{dim} \operatorname{ker} D_{\mathrm{APS}, \alpha}^{-}-\operatorname{dim} \operatorname{ker} D_{\mathrm{APS}, \alpha}^{+} .
\end{aligned}
$$

Together, (4) and (5) show that both inequalities in (5) must be equalities, which proves $(2)$.

We will need (2) to identify $\operatorname{codim}\left(\operatorname{Ran}\left(D_{\mathrm{APS}, \alpha}^{+}\right)\right)$with $\operatorname{dim} \operatorname{ker} D_{\mathrm{APS}, \alpha}^{-}$.

2.5. The Calderón projector. Let $\mathcal{C} \subset \mathcal{C}^{\infty}(M, \Sigma(M))$ be the image of the Calderón projector, or equivalently the space of boundary values of smooth solutions on $Y$ to the equation $D^{+} \phi=0$. For all $I \subset \mathbb{R}$, the unique continuation property of harmonic spinors shows that the map of restriction to $M$ induces an isomorphism

$$
\operatorname{ker}\left(D_{\mathrm{APS}, I}^{+}\right) \rightarrow \mathcal{C} \cap \operatorname{Ran}\left(\Pi_{I}\right) .
$$

For $J \subset I$ infinite intervals bounded below set

$$
\mathcal{C}_{I \backslash J}:=\mathcal{C} \cap \operatorname{Ran}\left(\Pi_{I}\right) / \mathcal{C} \cap \operatorname{Ran}\left(\Pi_{J}\right) .
$$

For example, $\mathcal{C}_{\{0\}}=\mathcal{C} \cap \operatorname{Ran}\left(\Pi_{[0, \infty)}\right) / \mathcal{C} \cap \operatorname{Ran}\left(\Pi_{(0, \infty)}\right)$ is the space $h_{\infty}$ of limiting values of extended $L^{2}$ solutions from [1, Corollary 3.14]. Clearly

$$
\operatorname{dim}\left(\mathcal{C}_{[\beta, \gamma)}\right) \leq N[\beta, \gamma) .
$$

Note that for $p=2 n /(n-1)$, Corollary 2 reads

$$
\operatorname{index}\left(D_{p, p^{\prime}, \text { cone }}^{+}\right)=\int_{X} \widehat{A}\left(g_{\text {cone }}\right)+\frac{1}{2} \eta_{[\alpha, \infty)}(M)-h_{\infty} .
$$

3. Step 1: Conformal change. To illustrate our method we first give the proof of Corollary 3 in the case $p=2$. The Dirac operator $D_{\text {cone }}^{ \pm}$: $\mathcal{C}^{\infty}\left(X, \Sigma^{ \pm}\right) \rightarrow \mathcal{C}^{\infty}\left(X, \Sigma^{\mp}\right)$ restricts to a densely defined unbounded operator

$$
D_{\text {cone }}^{ \pm}: \mathcal{C}_{\mathrm{c}}^{\infty}\left(X, \Sigma^{ \pm}\right) \subset L^{2}\left(X, \Sigma^{ \pm}, g_{\text {cone }}\right) \rightarrow L^{2}\left(X, \Sigma^{\mp}, g_{\text {cone }}\right) .
$$

Note that $\overline{D_{\text {cone }}^{+}}$is the operator $\bar{D}_{0}^{+}$of Chou [3].

Let $r: X \rightarrow(0, \infty)$ denote a smooth extension of the distance to the singularity. Consider the conformal metric

$$
g_{\text {cyl }}:=r^{-2} g_{\text {cone }} \text {. }
$$

The spinor bundles for the two metrics $g_{\text {cyl }}$ and $g_{\text {cone }}$ on $X$ are the same. The two Dirac operators are related by [5, Prop. 1.3]:

$$
D_{\text {cyl }}=r^{(n+1) / 2} D_{\text {cone }} r^{-(n-1) / 2}
$$

where $n$ is the dimension of $X$. Consider the isometry of Hilbert spaces

$$
L^{2}\left(X, \Sigma, g_{\text {cone }}\right) \rightarrow L^{2}\left(X, \Sigma, g_{\text {cyl }}\right), \quad \phi \mapsto r^{n / 2} \phi .
$$


Using (8), we see that the operator $D_{\text {cone }}^{ \pm}$from (6) is conjugated via the above isometry to

$$
r^{-1 / 2} D_{\text {cyl }}^{ \pm} r^{-1 / 2}: \mathcal{C}_{\mathrm{c}}^{\infty}\left(X, \Sigma^{ \pm}\right) \subset L^{2}\left(X, \Sigma^{ \pm}, g_{\mathrm{cyl}}\right) \rightarrow L^{2}\left(X, \Sigma^{\mp}, g_{\mathrm{cyl}}\right) .
$$

Use the change of variables

$$
t=-\log r+\log \varepsilon-1 .
$$

Then $g_{\text {cyl }}=d t^{2}+g_{M}$ for $t>-1$ so $\left(X, g_{\text {cyl }}\right)$ is complete with cylindrical ends. The equalities $(1)$ hold over the cylinder $(-1, \infty) \times M$. For simplicity, we define the operators

$$
D^{ \pm}:=e^{t / 2} D_{\text {cyl }}^{ \pm} e^{t / 2}=\frac{e}{\varepsilon} r^{-1 / 2} D_{\text {cyl }}^{ \pm} r^{-1 / 2}
$$

with domains given by $(9)$. Since $D_{\text {cone }}^{+}$and $(\varepsilon / e) D^{+}$are conjugated via an isometry, we have trivially

$$
\operatorname{index}\left(D_{\text {cone }}^{+}\right)=\operatorname{index}\left(D^{+}\right) .
$$

4. Step 2: Restriction to a finite cylinder. Let $Y \subset X$ be the compact manifold with boundary defined by $t \leq 0$. Let $\phi \in \operatorname{ker} D^{+*}$ and let

$$
\phi(t)=\sum_{\lambda \in \operatorname{Spec} D_{M}} a_{\lambda}(t) \phi_{\lambda}, \quad D_{M} \phi_{\lambda}=\lambda \phi_{\lambda},
$$

be the decomposition of $\phi$ over the cylinder in an orthonormal base of eigenspinors of $D_{M}$. From Lemma $8, D^{+*} \phi=0$ is equivalent to $D^{-} \phi=0$ in distributional sense. By elliptic regularity, $\phi$ is smooth. Using (1), over the cylinder $(-1, \infty) \times M$ we get

$$
\begin{aligned}
\left(D^{-} \phi\right)(t)=0 & \Leftrightarrow \sum_{\lambda \in \operatorname{Spec} D_{M}} e^{t / 2}\left(-\partial_{t}\left(e^{t / 2} a_{\lambda}(t)\right)+\lambda e^{t / 2} a_{\lambda}(t)\right) \phi_{\lambda}=0 \\
& \Leftrightarrow a_{\lambda}(t)=e^{(\lambda-1 / 2) t} a_{\lambda}(0), \forall \lambda \in \operatorname{Spec}\left(D_{M}\right) .
\end{aligned}
$$

Since $\phi \in L^{2}\left(X, \Sigma^{-}, g_{\text {cyl }}\right)$ we deduce that $a_{\lambda}(0)=0$ for all $\lambda \geq 1 / 2$. In other words, $\phi_{\mid Y} \in \mathcal{C}^{\infty}\left(Y, \Sigma^{-}, \Pi_{(-\infty, 1 / 2)}\right)$. Moreover, $\phi_{\mid Y}$ lives in the kernel of the partial differential operator $D^{-}$on $Y$.

Conversely, let $\phi_{Y} \in \mathcal{C}^{\infty}\left(Y, \Sigma^{-}, \Pi_{(-\infty, 1 / 2)}\right)$ be a solution to $D^{-} \phi_{Y}=0$. Then over the cylinder $(-1,0] \times M \subset Y$, the coefficients of $\phi_{Y}$ satisfy (12). The spectral condition at $t=0$ implies that $a_{\lambda}=0$ for $\lambda \geq 1 / 2$. Therefore we can consistently define $\phi \in \mathcal{C}^{\infty}\left(X, \Sigma^{-}\right)$by

$$
\phi= \begin{cases}\phi_{Y} & \text { over } Y, \\ \sum_{1 / 2>\lambda \in \operatorname{Spec} D_{M}} e^{(\lambda-1 / 2) t} a_{\lambda}(0) \phi_{\lambda} & \text { over }(-1, \infty) \times M .\end{cases}
$$

It is clear that $\phi$ is a distributional solution to $D^{-} \phi=0$ and moreover $\phi \in L^{2}\left(X, \Sigma^{-}, g_{\mathrm{cyl}}\right)$. For this last fact it is crucial that $\lambda<1 / 2$. Summarizing the above discussion, we proved 


\section{Proposition 13. Restriction to $Y$ induces an isomorphism}

$r_{Y}: \operatorname{ker} D^{+*} \rightarrow \operatorname{ker}\left(D_{\mathrm{APS}, 1 / 2}^{-}: \mathcal{C}^{\infty}\left(Y, \Sigma^{-}, \Pi_{(-\infty, 1 / 2)}\right) \rightarrow \mathcal{C}^{\infty}\left(Y, \Sigma^{+}\right)\right)$.

Similarly, let $\phi \in \operatorname{ker} D^{-*}$. Then over $(-1, \infty) \times M$ we have

$$
\phi(t)=\sum_{-1 / 2<\lambda \in \operatorname{Spec} D_{M}} e^{-(1 / 2+\lambda) t} a_{\lambda}(0) \phi_{\lambda} .
$$

As above, the restriction map induces an isomorphism

$$
r_{Y}: \operatorname{ker} D^{-*} \rightarrow \operatorname{ker}\left(D_{\mathrm{APS},(-1 / 2, \infty)}^{+}: \mathcal{C}^{\infty}\left(Y, \Sigma^{+}, \Pi_{(-1 / 2, \infty)}\right) \rightarrow \mathcal{C}^{\infty}\left(Y, \Sigma^{-}\right)\right) .
$$

We are actually interested in $\operatorname{ker} \overline{D^{+}}$, an operator which is smaller than $D^{-*}$, so $\operatorname{ker} \overline{D^{+}}=\operatorname{ker} D^{-*} \cap \operatorname{dom}\left(\overline{D^{+}}\right)$.

Lemma 14. Let $\phi \in \mathcal{C}^{\infty}\left(X, \Sigma^{+}\right)$satisfy $(13)$ over the cylinder $(-1, \infty)$ $\times M$. Then $\phi \in \operatorname{dom}\left(\overline{D^{+}}\right)$if and only if $a_{\lambda}(0)=0$ for all $\lambda<1 / 2$.

Proof. First assume that $a_{\lambda}(0)=0$ for all $\lambda<1 / 2$. Let $\chi: X \rightarrow[0,1]$ be a smooth cut-off function with the properties

$$
\chi(t):= \begin{cases}1 & \text { if } t<1 \\ 0 & \text { if } t>2\end{cases}
$$

For $k \in \mathbb{N}^{*}$ let $\chi_{k}(t):=\chi(t / k)$ and define $\psi_{k}:=\chi_{k} \phi \in \mathcal{C}_{\mathrm{c}}^{\infty}\left(X, \Sigma^{+}\right)$. Clearly, $\lim _{k \rightarrow \infty} \psi_{k}=\phi$ in $L^{2}$. Moreover,

$$
D^{+} \psi_{k}= \begin{cases}D^{+} \phi & \text { over } Y \\ k^{-1} \chi^{\prime}(t / k) e^{t} \phi & \text { over }(-1, \infty) \times M\end{cases}
$$

The assumption on $\lambda$ and (13) show that $\left|e^{t} \phi(t)\right|_{L^{2}(M, \Sigma(M))}$ is bounded as a function of $t$. Since $\int_{0}^{\infty} k^{-2} \chi^{\prime}(t / k)^{2} d t=C / k \rightarrow 0$ as $k \rightarrow \infty$, it follows that $D^{+} \psi_{k}$ converges in $L^{2}$ (to $D^{+} \phi$, a compactly supported smooth distribution) so $\phi \in \operatorname{dom}\left(\overline{D^{+}}\right)$.

Let now $\phi \in \operatorname{dom}\left(\overline{D^{+}}\right)$satisfy (13). Since $\chi \phi$ has compact support, it follows that $(1-\chi(t)) \phi \in \operatorname{dom}\left(\overline{D^{+}}\right)$. In the sense of distributions,

$$
D^{+}((1-\chi(t)) \phi)=-\chi^{\prime}(t) e^{t} \phi
$$

because (13) implies that $D^{+} \phi=0$ on the support of $1-\chi$. Thus there exists a sequence $\mathcal{C}_{\mathrm{c}}^{\infty}\left(X, \Sigma^{+}\right) \ni \psi_{k}$ such that for $k \rightarrow \infty$,

$$
\begin{gathered}
\psi_{k} \stackrel{L^{2}}{\rightarrow}(1-\chi(t)) \sum_{\substack{\lambda \in \operatorname{Spec} D_{M} \\
\lambda>-1 / 2}} e^{-(\lambda+1 / 2) t} a_{\lambda}(0) \phi_{\lambda}, \\
D^{+} \psi_{k} \stackrel{L^{2}}{\rightarrow} D^{-*}(1-\chi(t)) \phi=-\chi^{\prime}(t) \sum_{\substack{\lambda \in \operatorname{Spec} D_{M} \\
\lambda>-1 / 2}} e^{-(\lambda-1 / 2) t} a_{\lambda}(0) \phi_{\lambda} .
\end{gathered}
$$


The right-hand side is supported in $[1, \infty) \times M$, so the sequence $\left\{(1-\chi(t+1)) \psi_{k}\right\}$ also satisfies (14). Thus we may assume that $\psi_{k}$ is supported on $[0, \infty) \times M$. Let $\lambda \in(-1 / 2,1 / 2) \cap \operatorname{Spec}\left(D_{M}\right)$. We pair the second limit in (14) with the $L^{2}$ distribution

$$
u_{\lambda}= \begin{cases}0 & \text { on } X \backslash(-1, \infty) \times M, \\ a_{\lambda}(0) e^{(\lambda-1 / 2) t} \phi_{\lambda} & \text { on }(-1, \infty) \times M\end{cases}
$$

We get

$$
\lim _{k \rightarrow \infty}\left(D^{+} \psi_{k}, u_{\lambda}\right)=-\left|a_{\lambda}(0)\right|^{2} \int_{0}^{\infty} \chi^{\prime}(t) d t=\left|a_{\lambda}(0)\right|^{2} .
$$

On the other hand, by definition $\left(D^{+} \psi_{k}, u_{\lambda}\right)=\left(\psi_{k}, D^{+*} u_{\lambda}\right)=0$ since $D^{-} u_{\lambda}$ (in the sense of distributions) is supported at $t=-1$, thus outside the support of $\psi_{k}$. Therefore $a_{\lambda}(0)=0$.

Thus restriction to $Y$ gives an isomorphism

$$
r_{Y}: \operatorname{ker} \overline{D^{+}} \rightarrow \operatorname{ker}\left(D_{\mathrm{APS}, 1 / 2}^{+}: \mathcal{C}^{\infty}\left(Y, \Sigma^{+}, \Pi_{[1 / 2, \infty)}\right) \rightarrow \mathcal{C}^{\infty}\left(Y, \Sigma^{-}\right)\right) .
$$

Together with Proposition 13 and Corollary 12 we proved

$$
\operatorname{index}\left(D^{+}\right)=\operatorname{index}\left(D_{\mathrm{APS}, 1 / 2}^{+}\right) \text {. }
$$

5. Step 3: The $L^{2}$ index formula. Corollary 12 and Lemma 9 give

$$
\operatorname{index}\left(D_{\mathrm{APS}, 1 / 2}^{+}\right)=\int_{Y} \widehat{A}\left(g_{\mathrm{cyl}}\right)+\frac{1}{2} \widetilde{\eta}(M)-N[0,1 / 2) .
$$

We claim that

$$
\int_{Y} \widehat{A}\left(g_{\text {cyl }}\right)=\int_{X} \widehat{A}\left(g_{\text {cone }}\right) .
$$

Indeed, the $A$-hat form is a Pontryagin form; as such, it only involves the Weyl tensor and therefore it is conformally invariant. So $\widehat{A}\left(g_{\text {cyl }}\right)=\widehat{A}\left(g_{\text {cone }}\right)$. Moreover, $\widehat{A}\left(g_{\text {cyl }}\right)$ vanishes on the cylinder $(-1, \infty) \times M$ by multiplicativity, so $\widehat{A}\left(g_{\text {cone }}\right)$ also vanishes on the cone $X \backslash Y$. In particular, $\widehat{A}\left(g_{\text {cone }}\right)$ has compact support on $X$. Consequently,

$$
\begin{aligned}
\operatorname{index}\left(D_{\text {cone }}^{+}\right) & =\operatorname{index}\left(D^{+}\right) & & \text {by }(11) \\
& =\operatorname{index}\left(D_{\mathrm{APS}, 1 / 2}^{+}\right) & & \text {by }(15) \\
& =\int_{X} \widehat{A}\left(g_{\text {cone }}\right)+\frac{1}{2} \widetilde{\eta}(M)-N[0,1 / 2) & & \text { by Cor. } 12 .
\end{aligned}
$$

This is Chou's formula [3, Thm. 5.23]. 
6. The $L^{p} \rightarrow L^{q}$ index. For $1<p \in \mathbb{R}$, let $L^{p}\left(X, \Sigma^{ \pm}, g_{\text {cone }}\right)$ be the Banach space of $p$-integrable spinors obtained by completing $\mathcal{C}_{\mathrm{c}}^{\infty}\left(X, \Sigma^{ \pm}\right)$in the $L^{p}$ norm:

$$
\|\phi\|_{L^{p}}^{p}:=\int_{X}|\phi|^{p} g_{\text {cone }} .
$$

Let $p^{\prime} \in(1, \infty)$ be the "dual" of $p$, i.e., $1 / p+1 / p^{\prime}=1$ so that integration on $X$ gives a bilinear pairing

$$
L^{p}\left(X, \Sigma^{ \pm}, g_{\text {cone }}\right) \times L^{p^{\prime}}\left(X, \Sigma^{ \pm}, g_{\text {cone }}\right) \rightarrow \mathbb{C} .
$$

It is well known that $L^{p}$ is reflexive, so the above pairing identifies the space $L^{p}\left(X, \Sigma^{ \pm}, g_{\text {cone }}\right)^{\prime}$ with $L^{p^{\prime}}\left(X, \Sigma^{ \pm}, g_{\text {cone }}\right)$. The main result of this paper is Theorem 1, the computation of the index of

$$
D_{p, q, \text { cone }}^{+}: \mathcal{C}_{\mathrm{c}}^{\infty}\left(X, \Sigma^{+}\right) \subset L^{p}\left(X, \Sigma^{+}, g_{\text {cone }}\right) \rightarrow L^{q}\left(X, \Sigma^{-}, g_{\text {cone }}\right)
$$

in the sense of Definition 5 , for $p, q>1$.

Proof of Theorem 1. We follow the strategy already used above for $p=$ $q=2$. First we conjugate $D_{p, q, \text { cone }}^{+}$to an operator acting in the cylindrical $L^{p}$ spaces, where $g_{\text {cyl }}$ is given by $(7)$ :

$$
\begin{array}{ccc}
L^{p}\left(X, \Sigma^{+}, g_{\text {cone }}\right) & \stackrel{D_{p, q, \text { cone }}^{+}}{\longrightarrow} & L^{q}\left(X, \Sigma^{-}, g_{\text {cone }}\right) \\
r^{n / p} \downarrow & & r^{n / q .} \downarrow \\
L^{p}\left(X, \Sigma^{+}, g_{\text {cyl }}\right) & \stackrel{c D^{+}}{\longrightarrow} & L^{q}\left(X, \Sigma^{-}, g_{\text {cyl }}\right)
\end{array}
$$

with $c=(e / \varepsilon)^{\alpha_{1}+\alpha_{2}}$. The vertical arrows are isometries. Using (8) we see that

$$
D^{+}=\left(\frac{\varepsilon}{e}\right)^{\alpha_{1}+\alpha_{2}} r^{-\alpha_{2}} D_{p, q, \mathrm{cyl}}^{+} r^{-\alpha_{1}}=e^{\alpha_{2} t} D_{p, q, \mathrm{cyl}}^{+} e^{\alpha_{1} t}
$$

after the coordinate change (10). As in the $L^{2}$ case, it is obvious that

$$
\operatorname{index}\left(D_{p, \text { cone }}^{+}\right)=\operatorname{index}\left(D^{+}\right) \text {. }
$$

Note that the formal adjoint

$$
D^{-}: \mathcal{C}_{\mathrm{c}}^{\infty}\left(X, \Sigma^{-}\right) \subset L^{q^{\prime}}\left(X, \Sigma^{-}, g_{\text {cyl }}\right) \rightarrow L^{p^{\prime}}\left(X, \Sigma^{-}, g_{\text {cone }}\right)
$$

is given by

$$
D^{-}=e^{\alpha_{1} t} D_{p, q, \mathrm{cyl}}^{-} e^{\alpha_{2} t} .
$$

Let $\phi \in \operatorname{ker} D^{+*}$, so by Lemma $8, \phi \in L^{q^{\prime}}\left(X, \Sigma^{-}, g_{\text {cyl }}\right)$ is a distributional solution of $D^{-}$. By elliptic regularity, $\phi$ is smooth. Using (1), we see that the restriction of $\phi$ to the cylinder $\{t \geq 0\}$ is explicitly given by the analog of (12):

$$
\phi(t)=\sum_{\lambda \in \operatorname{Spec} D_{M}} e^{\left(\lambda-\alpha_{2}\right) t} a_{\lambda}(0) \phi_{\lambda} .
$$


Lemma 15. Fix $w \in(1, \infty)$. A smooth spinor $\phi$ which satisfies (17) belongs to $L^{w}\left(X, \Sigma^{-}, g_{\text {cyl }}\right)$ if and only if $a_{\lambda}(0)=0$ for all $\lambda \geq \alpha_{2}$.

Proof. This is clear for $w=2$ but in general it needs a proof. First assume that $a_{\lambda}(0)=0$ for all $\lambda \geq \alpha_{2}$. Then the $L^{2}$ Sobolev norms of $\phi(t)$ decrease exponentially with $t$. More precisely, let $\|\phi(t)\|_{H^{k}(M)}:=\left\|D_{M}^{k} \phi(t)\right\|_{L^{2}(M)}$. Since Spec $D_{M}$ is discrete, there exists $\varepsilon>0$ such that $\alpha_{2}-\lambda>\varepsilon$ for all $\lambda \in \operatorname{Spec} D_{M} \cap\left(-\infty, \alpha_{2}\right)$. Then

$$
\|\phi(t)\|_{H^{k}(M)}<C_{k} e^{-\varepsilon t} .
$$

By the Sobolev embeddings, the $C^{0}$ norm of $\phi(t)$ also decreases exponentially, so $\phi \in L^{w}$ for all $w \geq 1$.

Conversely, assume that $a_{\lambda}(0) \neq 0$ for some $\lambda \geq \alpha_{2}$. We can assume that $a_{\lambda}(0)=1$. Let $C:=\left\|\phi_{\lambda}\right\|_{L^{\infty}(M, \Sigma(M))}$. Then

$$
C\|\phi(t)\|_{L^{1}(M, \Sigma(M))} \geq \int_{M}\left(\phi(t), \phi_{\lambda}\right) d x e^{\left(\lambda-\alpha_{2}\right) t} \geq 1
$$

so for $w^{\prime}$ the "dual" of $w$,

$$
\int_{M}|\phi(t)|^{w} d x \geq C^{-w} \operatorname{Vol}(M)^{-w / w^{\prime}}
$$

and therefore $|\phi|^{w}$ is not integrable on the cylinder.

Recall that $Y$ is the compact manifold with boundary obtained from $X$ after removing the cylinder $(0, \infty) \times M$. We just proved that the restriction to $Y$ defines an isomorphism

$$
\operatorname{ker} D^{+*} \simeq \operatorname{ker} D_{\mathrm{APS}, \alpha_{2}}^{-} \text {. }
$$

Similarly, $\phi \in \operatorname{ker} D^{-*}$ is equivalent to $\phi \in \mathcal{C}^{\infty}\left(X, \Sigma^{+}\right), D^{+} \phi=0$ in the sense of distributions, and the restriction of $\phi$ to the cylinder satisfies

$$
\phi(t)=\sum_{\substack{\lambda \in \operatorname{Spec} D_{M} \\ \lambda>-\alpha_{1}}} e^{-\left(\lambda+\alpha_{1}\right) t} a_{\lambda}(0) \phi_{\lambda} .
$$

We deduce that restriction to $Y$ gives the isomorphism

$$
r_{Y}: \operatorname{ker} D^{-*} \rightarrow \operatorname{ker}\left(D_{\mathrm{APS},\left(-\alpha_{1}, \infty\right)}^{+}\right) \text {. }
$$

We need to decide which elements in $\operatorname{ker} D^{-*}$ live in $\operatorname{dom}\left(\overline{D^{+}}\right)$. The series (19) clearly converges in $L^{2}$, but we need to add to the proof of Lemma 14 the argument for $L^{p}$ convergence. For the sake of clarity we give again the full proof.

Lemma 16. Let $\phi \in \mathcal{C}^{\infty}\left(X, \Sigma^{+}\right)$be of the form (19) over the cylinder. Then $\phi \in \operatorname{dom}\left(\overline{D^{+}}\right)$if and only if the coefficients of $(19)$ satisfy $a_{\lambda}(0)=0$ for all $\lambda<\alpha_{2}$. 
Proof. Assume that $a_{\lambda}(0)=0$ for all $\lambda<\alpha_{2}$. By the Sobolev embedding theorem we prove as in Lemma 15 that for some $\varepsilon>0$,

$$
|\phi(t, x)|< \begin{cases}C e^{-\left(\varepsilon+\alpha_{1}+\alpha_{2}\right) t} & \text { for } \alpha_{1}+\alpha_{2}>0, \\ C e^{-\varepsilon t} & \text { for } \alpha_{1}+\alpha_{2} \leq 0,\end{cases}
$$

so in particular $\phi \in L^{p}$. Use the functions $\chi, \chi_{k}$ from the proof of Lemma 14. The inequality $(21)$ shows that $\chi_{k} \phi \stackrel{L^{p}}{\rightarrow} \phi$. Then $D^{+}\left(\chi_{k} \phi\right)=\chi_{k} D^{+} \phi+$ $\chi_{k}^{\prime}(t) e^{\left(\alpha_{1}+\alpha_{2}\right) t} \phi$. Clearly $\chi_{k} D^{+} \phi=D^{+} \phi$ since $\chi_{k}$ equals 1 on the support of $D^{+} \phi$. Again by (21), the $L^{q}$ norm of $e^{\left(\alpha_{1}+\alpha_{2}\right) t} \phi(t)$ is bounded in $t$ and so by changing variables,

$$
\left.\int_{X} \chi_{k}^{\prime}(t) e^{\left(\alpha_{1}+\alpha_{2}\right) t} \phi(t)\right|^{q} d x d t \leq \frac{C}{k^{q-1}} .
$$

This implies that $D^{+}\left(\chi_{k} \phi\right) \stackrel{L^{q}}{\rightarrow} D^{+} \phi$ as $k \rightarrow \infty$.

For the converse, there is nothing to prove if $\alpha_{2} \leq-\alpha_{1}$; therefore assume $\alpha_{1}+\alpha_{2}>0$. Let $\phi \in \operatorname{dom}\left(\overline{D^{+}}\right)$. Since $\chi(t) \phi \in \operatorname{dom}\left(D^{+}\right)$it follows that $(1-\chi(t)) \phi \in \operatorname{dom}\left(\overline{D^{+}}\right)$. By definition, there exists a sequence $\left\{\psi_{k}\right\}_{k \in \mathbb{N}}$ of compactly supported spinors such that

$$
\begin{gathered}
\psi_{k} \stackrel{L^{p}}{\rightarrow}(1-\chi(t)) \sum_{\substack{\lambda \in \operatorname{Spec} D_{M} \\
\lambda>-\alpha_{1}}} e^{-\left(\lambda+\alpha_{1}\right) t} a_{\lambda}(0) \phi_{\lambda}, \\
D^{+} \psi_{k} \stackrel{L^{q}}{\rightarrow} D^{-*}((1-\chi(t)) \phi)=-\chi^{\prime}(t) \sum_{\substack{\lambda \in \operatorname{Spec} D_{M} \\
\lambda>-\alpha_{1}}} e^{-\left(\lambda-\alpha_{2}\right) t} a_{\lambda}(0) \phi_{\lambda} .
\end{gathered}
$$

The sequence $\left\{(1-\chi(t+1)) \psi_{k}\right\}$ also satisfies (22), so we can assume that $\operatorname{supp}\left(\psi_{k}\right) \subset[0, \infty) \times M$.

For $\lambda \in\left(-\alpha_{1}, \alpha_{2}\right)$ consider the distribution

$$
u_{\lambda}= \begin{cases}0 & \text { on } X \backslash(-1, \infty) \times M, \\ a_{\lambda}(0) e^{\left(\lambda-\alpha_{2}\right) t} \phi_{\lambda} & \text { on }(-1, \infty) \times M .\end{cases}
$$

Since $\lambda<\alpha_{2}$, it follows that $u_{\lambda} \in L^{p}$ so the second limit in (22) commutes with the pairing with $u_{\lambda}$ :

$$
\lim _{k \rightarrow \infty}\left(D^{+} \psi_{k}, u_{\lambda}\right)=-\left|a_{\lambda}(0)\right|^{2} \int_{0}^{\infty} \chi^{\prime}(t) d t=\left|a_{\lambda}(0)\right|^{2} .
$$

On the other hand, by definition

$$
\left(D^{+} \psi_{k}, u_{\lambda}\right)=\left(\psi_{k}, D^{+*} u_{\lambda}\right)=0
$$

since $D^{-} u_{\lambda}$ (in the sense of distributions) is supported at $t=-1$, thus outside the support of $\psi_{k}$. Therefore $a_{\lambda}(0)=0$. 
By Lemma 16 and (20),

$$
\operatorname{ker} \overline{D_{p, \text { cone }}^{+}} \simeq \begin{cases}\operatorname{ker}\left(D_{\mathrm{APS}, \alpha_{2}}^{+}\right) & \text {for } \alpha_{1}+\alpha_{2}>0 \\ \operatorname{ker}\left(D_{\mathrm{APS},\left(-\alpha_{1}, \infty\right)}^{+}\right) & \text {for } \alpha_{1}+\alpha_{2} \leq 0\end{cases}
$$

In the second case,

$$
\operatorname{dim} \operatorname{ker} \overline{D^{+}}=\operatorname{dim} \operatorname{ker} D_{\mathrm{APS}, \alpha_{2}}^{+}-\mathcal{C}_{\left[\alpha_{2},-\alpha_{1}\right]}
$$

because by definition ker $D_{\mathrm{APS},\left(-\alpha_{1}, \infty\right)}^{+} \simeq \mathcal{C}_{\left(-\alpha_{1}, \infty\right)}$. Recall now from Section 5 that the $\widehat{A}$ form is a conformal invariant and vanishes near the singularity. These facts together with (18), (16) and Corollary 12 finish the proof of Theorem 1.

7. Fictitious conical singularities. Let $(\bar{X}, g)$ be a closed spin manifold which contains a finite set $\{O\}$ of Euclidean points, in the sense that each $O_{j} \in\{O\}$ has a flat neighborhood. Writing $g$ in polar coordinates near $O_{j}$, we see that $X:=\bar{X} \backslash\{O\}$ is a conical spin manifold, and the basis of the cone is a disjoint union of spheres with the standard metric. For $n \geq 3$ the sphere $S^{n-1}$ has a unique spin structure, while for $n=2$ the spin structure on each circle must be bounding (non-trivial). The eigenvalues of the associated Dirac operator $D_{S^{n-1}}$ are

$$
\pm\left(\frac{n-1}{2}+k\right), \quad k=0,1,2, \ldots,
$$

with multiplicity $2^{[(n-1) / 2]}\left(\begin{array}{c}k+n-2 \\ k\end{array}\right)$ (see, e.g., [2]). The eigenspinors for the smallest eigenvalues $\pm(n-1) / 2$ are simply restrictions of parallel (i.e., constant) positive, respectively negative spinors from $\mathbb{R}^{n}$. In particular there is no eigenvalue between 0 and $1 / 2$. So for the $L^{2}$ index, Chou's formula reproved in Section 5 and the Atiyah-Singer formula give

$$
\operatorname{index}_{L^{2}}\left(D_{\text {cone }}^{+}\right)=\operatorname{index}\left(D_{\bar{X}}^{+}\right) \text {. }
$$

This equality may come as a surprise, since the domains of the two operators are not the same. Moreover, a similar equality is not true for the index of $D_{p, q, \text { cone }}^{+}$! This can be seen by simply comparing the index formulae. We discuss below the case of $D_{p, p^{\prime}, \text { cone }}^{+}$.

Proposition 17. For $p \geq n /(n-1)$ every spinor in the nullspace of $\overline{D_{p, p^{\prime}, \text { cone }}^{+}}$, respectively $D_{p, p^{\prime}, \text { cone }}^{+, * x t e n d s ~ t o ~ a ~ h a r m o n i c ~ s p i n o r ~ o n ~} \bar{X}$. Conversely, the restriction of every harmonic spinor on $\bar{X}$ to $X$ belongs to the nullspace of $\overline{D_{p, p^{\prime}, \text { cone }}^{+}}$, respectively $D_{p, p^{\prime}, \text { cone }}^{+, *}$.

Proof. First notice that $\alpha_{1}=\alpha_{2}=: \alpha$ satisfies

$$
-\frac{n-1}{2}<\alpha<\frac{n+1}{2} \text {. }
$$


The only eigenvalue of $D_{S^{n-1}}$ in this interval is $(n-1) / 2$, with multiplicity $2^{[(n-1) / 2]}$. Then from $(23)$,

$$
\operatorname{ker} \overline{D_{p, p^{\prime}, \text { cone }}^{+}} \simeq \begin{cases}\operatorname{ker}\left(D_{\mathrm{APS},(n-1) / 2}^{+}\right) & \text {if } \alpha \leq(n-1) / 2 \\ \operatorname{ker}\left(D_{\mathrm{APS},(n+1) / 2}^{+}\right) & \text {if } \alpha>(n-1) / 2\end{cases}
$$

and from (18),

$$
\operatorname{ker} D_{p, p^{\prime}, \text { cone }}^{+, *} \simeq \begin{cases}\operatorname{ker}\left(D_{\mathrm{APS},(-\infty,-(n-1) / 2]}^{-}\right) & \text {if } \alpha \leq(n-1) / 2, \\ \operatorname{ker}\left(D_{\mathrm{APS},(-\infty,(n-1) / 2]}^{-}\right) & \text {if } \alpha>(n-1) / 2\end{cases}
$$

Assume now that $\alpha \leq(n-1) / 2$, or equivalently $p \geq n /(n-1)$. Let $\phi_{Y}$ be a harmonic spinor in $\operatorname{ker}\left(D_{\mathrm{APS},(n-1) / 2}^{+}\right)$. From (19),

$$
\phi_{Y}(t)=\sum_{\substack{\lambda \in \operatorname{Spec} D_{S} n-1 \\ \lambda \geq(n-1) / 2}} e^{-(\lambda+\alpha) t} \phi_{\lambda}
$$

for $-1<t \leq 0$, where $\phi_{\lambda}$ is an eigenspinor of eigenvalue $\lambda$ but not necessarily of $L^{2}$ length 1 . Extend $\phi_{Y}$ to $X$ by the same formula for $t>0$ and then pull it back to a spinor $\phi_{\text {cone }}$ on the cone via the isometry from the proof of Theorem 1. Therefore

$$
\phi_{\text {cone }}(r)=r^{-n / p} \sum_{\substack{\lambda \in \operatorname{Spec} D_{S} n-1 \\ \lambda \geq(n-1) / 2}} e^{-(\lambda+\alpha) t} \phi_{\lambda}=\sum_{k=0}^{\infty} r^{k} \phi_{k+(n-1) / 2}
$$

so $\phi_{\text {cone }}$ extends to an $L^{\infty}$ spinor $\phi$ on $\bar{X}$. Now $\phi_{(n-1) / 2}$ is the restriction of a constant positive spinor from $\bar{X}$ to $S^{n-1}$, so it extends smoothly in $r=0$. From (8), $D_{\bar{X}}^{+} \sum_{k=1}^{\infty} r^{k} \phi_{k+(n-1) / 2}$ is also in $L^{\infty}\left(\bar{X}, \Sigma^{-}\right)$. Therefore the distribution $D_{\bar{X}}^{+} \phi$ is on one hand in $L^{\infty}(\bar{X})$ and on the other hand it vanishes on $X$. It follows that $\phi$ is a solution to $D_{\bar{X}}^{+}$.

Conversely, every solution $\phi$ to $D_{\bar{X}}^{+}$restricts to an $L^{p}$ distributional solution $\phi_{\text {cone }}$ to $D_{\text {cone }}^{+}$on $X$. Now $(20)$, Lemma 16 and the condition on $\alpha$

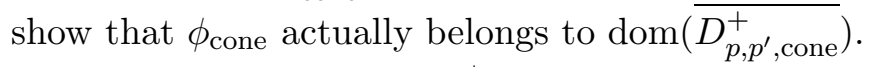

The statement about $D_{p, p^{\prime}, \text { cone }}^{+, *}$ is proved in the same way.

In conclusion, for $p \geq n /(n-1)$ the $L^{p}$ index problem on $X$ reduces to the usual index problem on $\bar{X}$. For $1<p<n /(n-1)$ the eigenvalue $(n-1) / 2$ of the Dirac operator on the sphere creeps into the picture, so the $L^{p}$ index of $D^{+}$on $X$ is $2^{[(n-1) / 2]}$ less than $\widehat{A}(\bar{X})$.

8. Possible extensions. Our goal was to give the simplest possible solution to the $L^{p}$ index problem, so we did not cover metrics which are only asymptotically conical. Our elementary method clearly breaks down in 
this case, and more conceptual approaches are needed, like parametrices for cone operators. Such parametrices can be constructed via either Melrose's bcalculus [6] or the cone calculus of Schulze [8]. General elliptic cone operators in $L^{p}$ spaces are treated in [7]. But an index formula generalizing Theorem 1 is still missing.

We have not discussed Fredholmness of our operators for the same reason, but note that the Fredholm property of $D_{p, p \text {,cone }}^{+}$seems to follow from the results of [7].

\section{References}

[1] M. F. Atiyah, V. K. Patodi and I. M. Singer, Spectral asymmetry and Riemannian geometry. I, Math. Proc. Cambridge Philos. Soc. 77 (1975), 43-69.

[2] C. Bär, The Dirac Operator on Space Forms of Positive Curvature, J. Math. Soc. Japan 48 (1996), 69-83.

[3] A. W. Chou, The Dirac operator on spaces with conical singularities and positive scalar curvatures, Trans. Amer. Math. Soc. 289 (1985), 1-40.

[4] N. Dines and B. W. Schulze, Mellin-edge representation of elliptic operators, Math. Methods Appl. Sci. 28 (2005), 2133-2172.

[5] N. Hitchin, Harmonic spinors, Adv. Math. 14 (1974), 1-55.

[6] R. B. Melrose, The Atiyah-Patodi-Singer Index Theorem, Res. Notes in Math. 4, A. K. Peters, Wellesley, MA, 1993.

[7] E. Schrohe and J. Seiler, Ellipticity and invertibility in the cone algebra on $L_{p}$-Sobolev spaces, Integral Equations Operator Theory 41 (2001), 93-114.

[8] B. W. Schulze, Pseudo-Differential Boundary Value Problems, Conical Singularities, and Asymptotics, Math. Topics 4, Akademie-Verlag, Berlin, 1994.

UFR MIG

Université Paul Sabatier

118 route de Narbonne

31062 Toulouse, France

E-mail: legrand@picard.ups-tlse.fr
Institutul de Matematică al Academiei Române P.O. Box 1-764

RO-014700 Bucureşti, Romania E-mail: moroianu@alum.mit.edu

Received November 17, 2004

Revised version September 26, 2006 